\title{
AGILE observations of PSR B1509-58: a new class of highly magnetized, 'soft' gamma-ray pulsars?
}

\author{
Maura Pilia ${ }^{* \dagger}$ \\ Dipartimento di Fisica, Università dell'Insubria, via Valleggio 11, I-22100, Como, Italy \\ E-mail: mpilia@oa-cagliari.inaf.it
}

\author{
Alberto Pellizzoni \\ INAF-Osservatorio Astronomico di Cagliari, località Poggio dei Pini, strada 54, I-09012 \\ Capoterra, Italy \\ E-mail: apellizz@oa-cagliari.inaf.it
}

\begin{abstract}
We present the results of $\sim 2.5$ years AGILE observations of PSR B1509-58. The modulation significance of the light-curve above $30 \mathrm{MeV}$ is at a $5 \sigma$ confidence level and the light-curve is similar to those found earlier up to $30 \mathrm{MeV}$ by COMPTEL: a broad asymmetric first peak reaching its maximum $0.39 \pm 0.02$ cycles after the radio peak plus a second peak at $0.94 \pm 0.03$. The gamma-ray spectral energy distribution of pulsed flux is well described by a power-law (photon index $\alpha=1.87 \pm 0.09$ ) with a remarkable cutoff at $E_{c}=81 \pm 20 \mathrm{MeV}$, representing the softest spectrum observed among $\gamma$-ray pulsars so far. The unusual soft break in the spectrum of PSR B1509-58 has been interpreted in the framework of polar cap models as a signature of the exotic photon splitting process in the strong magnetic field of this pulsar. In the case of an outergap scenario, or the two pole caustic model, better constraints on the geometry of the emission would be needed from the radio band in order to establish whether the conditions required by the models to reproduce AGILE light-curves and spectra match the polarization measurements.
\end{abstract}

25th Texas Symposium on Relativistic Astrophysics - TEXAS 2010

December 06-10, 2010

Heidelberg, Germany

\footnotetext{
*Speaker.

${ }^{\dagger}$ On behalf of the AGILE Team and AGILE Pulsar Working Group.
} 


\section{Introduction}

PSR B1509-58 was discovered as an X-ray pulsar with the Einstein satellite and soon also detected at radio frequencies (Manchester et al. 1982), with a derived distance supporting the association with the SNR MSH 15-52 $(d \sim 5.2 \mathrm{kpc})$. With a period $P \simeq 150 \mathrm{~ms}$ and a period derivative $\dot{P} \simeq 1.53 \times 10^{-12} \mathrm{~s} \mathrm{~s}^{-1}$, assuming the standard dipole vacuum model, the estimated spindown age for this pulsar is 1570 years and its inferred surface magnetic fi eld is one of the highest observed for an ordinary radio pulsar: $B=3.1 \times 10^{13} \mathrm{G}$, as calculated at the pole. Its rotational energy loss rate is $\dot{E}=1.8 \times 10^{37} \mathrm{erg} / \mathrm{s}$.

The young age and the high rotational energy loss rate made this pulsar a promising target for the gamma-ray satellites. In fact, the instruments on board of the Compton Gamma-Ray Observatory (CGRO) observed its pulsation at low gamma-ray energies, but it was not detected with high signifi cance by the Energetic Gamma-Ray Experiment Telescope (EGRET), the instrument operating at the energies from $30 \mathrm{MeV}$ to $30 \mathrm{GeV}$. This was remarkable, since all other known gamma-ray pulsars show spectral turnovers well above $100 \mathrm{MeV}$ (e.g. Thompson 2004). Harding et al. (1997) suggested that the break in the spectrum could be interpreted as due to inhibition of the pair-production caused by the photon-splitting phenomenon (Adler et al. 1970). The photon splitting appears, in the frame of the polar cap models, in relation with a very high magnetic fi eld. An alternative explanation is proposed by Zhang \& Cheng (2000) using a three dimensional outer gap model. They propose that the gamma-ray emission is produced by synchrotron-self Compton radiation above the outer gap.

The Italian satellite AGILE (Tavani et al. 2009) obtained the fi rst detection of PSR B1509-58 in the EGRET band (Pellizzoni et al. 2009b) confi rming the occurrence of a spectral break. Here we summarize the results of a $\sim 2.5 \mathrm{yr}$ monitoring campaign of PSR B1509-58 with AGILE, improving counts statistics, and therefore ligh-tcurve characterization, with respect to earlier AGILE observations. More details on this analysis can be found in Pilia et al. (2010). With these observations the spectral energy distribution (SED) at $E<300 \mathrm{MeV}$, where the remarkable spectral turnover is observed, can be assessed.

\section{AGILE Observations, Data Analysis and Results}

AGILE devoted a large amount of observing time to the region of PSR B1509-58. For details on AGILE observing strategy, timing calibration and gamma-ray pulsars analysis the reader can refer to Pellizzoni et al. (2009a,b). A total exposure of $3.8 \times 10^{9} \mathrm{~cm}^{2} \mathrm{~s}(E>100 \mathrm{MeV})$ was obtained during the $2.5 \mathrm{yr}$ period of observations (July 2007 - October 2009) which, combined with AGILE effective area, gives our observations a good photon harvest from this pulsar.

Simultaneous radio observations of PSR B1509-58 with the Parkes radiotelescope in Australia are ongoing since the epoch of AGILE's launch. Strong timing noise was present and it was accounted for using the fitwaves technique developed in the framework of the TEMPO2 radio timing software (Hobbs et al. 2004, 2006). Using the radio ephemeris provided by the Parkes telescope, we performed the folding of the gamma-ray light-curve including the wave terms (Pellizzoni et al. 2009a). An optimized analysis followed, aimed at cross-checking and maximization of the signifi cance of the detection, including an energy-dependent events extraction angle around 
the source position based on the instrument point-spread-function (PSF). The chi-squared $\left(\chi^{2}\right)$-test applied to the 10 bin light-curve at $E>30 \mathrm{MeV}$ gave a detection signifi cance of $\sigma=4.8$. The unbinned $Z_{n}^{2}$-test gave a signifi cance of $\sigma=5.0$ with $n=2$ harmonics. The difference between the radio and gamma-ray ephemerides was $\Delta P_{\text {radio }, \gamma}=10^{-9} \mathrm{~s}$, at a level lower than the error in the parameter, showing perfect agreement among radio and gamma-ray ephemerides as expected, further supporting our detection and AGILE timing calibration.

We observed PSR B1509-58 in three energy bands: 30-100 MeV, 100-500 MeV and above $500 \mathrm{MeV}$. We did not detect pulsed emission at a signifi cance $\sigma \geq 2$ for $E>500 \mathrm{MeV}$. The $\gamma$-ray light-curves of PSR B1509-58 for different energy bands are shown in Fig. 1. The AGILE E $>30$ $\mathrm{MeV}$ light-curve shows two peaks at phases $\phi_{1}=0.39 \pm 0.02$ and $\phi_{2}=0.94 \pm 0.03$ with respect to the single radio peak, here put at phase 0 . The phases are calculated using a Gaussian fi to the peaks, yielding a FWHM of $0.29(6)$ for the fi rst peak and of 0.13(7) for the second peak, where we quote in parentheses (here and throughout the paper) the $1 \sigma$ error on the last digit. The fi rst peak is coincident in phase with COMPTEL's peak (Kuiper et al. 1999). In its highest energy band (10-30 MeV) COMPTEL showed the indication of a second peak (even though the modulation had low signifi cance, $2.1 \sigma$ ). This second peak is coincident in phase with AGILE's second peak (Fig. 1). AGILE thus confi rms the previously marginal detection of a second peak.

Based on our exposure we derived the $\gamma$-ray flux from the number of pulsed counts. The pulsed fluxes in the three AGILE energy bands were $F_{\gamma}=10(4) \times 10^{-7} \mathrm{ph} \mathrm{cm}^{-2} \mathrm{~s}^{-1}$ in the 30 $100 \mathrm{MeV}$ band, $F_{\gamma}=2.1(5) \times 10^{-7} \mathrm{ph} \mathrm{cm}^{-2} \mathrm{~s}^{-1}$ in the $100-500 \mathrm{MeV}$ band and a $1 \sigma$ upper limit $F_{\gamma}<8 \times 10^{-8} \mathrm{ph} \mathrm{cm}^{-2} \mathrm{~s}^{-1}$ for $E>500 \mathrm{MeV}$.

Fig. 2 shows the SED of PSR B1509-58 based on AGILE's and COMPTEL's observed fluxes. Fermi upper limits are also shown, which are consistent with our measurements at a $2 \sigma$ confi dence level. COMPTEL observed this pulsar in three energy bands: $0.75-3 \mathrm{MeV}, 3-10 \mathrm{MeV}, 10-30 \mathrm{MeV}$, suggesting a spectral break between 10 and $30 \mathrm{MeV}$. AGILE pulsed flux confi rms the presence of a soft spectral break. As shown in Fig. 2, we modeled the observed COMPTEL and AGILE fluxes with a power-law plus cutoff fi $t$ using the Minuit minimization package (James et al. 1975): $F(E)=k \times E^{-\alpha} \exp \left[-\left(E / E_{c}\right)^{\beta}\right]$, with three free parameters: the normalization $k$, the spectral index $\alpha$, the cutoff energy $E_{c}$ and allowing $\beta$ to assume values of 1 and 2 (indicating either an exponential or a superexponential cutoff). No acceptable $\chi^{2}$ values were obtained for a superexponential cutoff, the presence of which can be excluded at a $3.5 \sigma$ confi dence level, while for an exponential cutoff we found $\chi_{v}^{2}=3.2$ for $v=2$ degrees of freedom, corresponding to a null hypothesis probability of 0.05 . The best values thus obtained for the parameters of the fi t were: $k=1.0(2) \times 10^{-4} \mathrm{~s}^{-1} \mathrm{~cm}^{-2}$, $\alpha=1.87(9), E_{c}=81(20) \mathrm{MeV}$.

\section{Discussion}

The bulk of the spin-powered pulsar flux is usually emitted in the MeV-GeV energy band with spectral breaks at $\leq 10 \mathrm{GeV}$ (e.g. Abdo et al. 2010a). PSR B1509-58 has the softest spectrum observed among gamma-ray pulsars, with a sub-GeV cutoff at $E \approx 80 \mathrm{MeV}$. In the following we discuss how the new AGILE observations can constrain the models for emission from the pulsar magnetosphere (for an extended discussion see Pilia et al. 2010). 
When PSR B1509-58 was detected in soft gamma-rays but not signifi cantly at $E>30 \mathrm{MeV}$, it was proposed that the mechanism responsible for this low-energy spectral break might be photon splitting (Harding et al. 1997). The photon splitting (Adler et al. 1970) is an exotic third-order quantum electro-dynamics process expected when the magnetic fi eld approaches or exceeds the critical value defi ned as $B_{c r}=m_{e}^{2} c^{3} /(e \hbar)=4.413 \times 10^{13} \mathrm{G}$. Most current theories for the generation of coherent radio emission in pulsar magnetospheres require formation of an electron-positron pair plasma developing via electromagnetic cascades. In very high magnetic fi elds the formation of pair cascades can be altered by the process of photon splitting: $\gamma \rightarrow \gamma \gamma$, which will operate as an attenuation mechanism in the high-fi eld regions near pulsar polar caps. Since it has no energy threshold, photon splitting can attenuate photons below the threshold for pair production, thus determining a spectral cutoff at lower energies.

In the case of PSR B1509-58 a polar cap model with photon splitting would be able to explain the soft gamma-ray emission and the low energy spectral cutoff, now quantifi ed by AGILE observations. Based on the observed cutoffs, which are related to the photons' saturation escape energy, we can derive constraints on the magnetic fi eld strength at emission, in the framework of photon splitting:

$$
\varepsilon_{e s c}^{s a t} \simeq 0.077\left(B^{\prime} \sin \theta_{k B, 0}\right)^{-6 / 5}
$$

where $\varepsilon_{e s c}^{\text {sat }}$ is the photon saturation escape energy, $B^{\prime}=B / B_{c r}$ and $\theta_{k B, 0}$ is the angle between the photon momentum and the magnetic fi eld vectors at the surface and is here assumed to be very small: $\theta_{k B, 0} \lesssim 0.57^{\circ}$ (see Harding et al. 1997). Using the observed energy cutoff ( $\varepsilon_{\text {esc }}^{\text {sat }} \simeq E=$ $80 \mathrm{MeV}$ ) we fi nd that $B \gtrsim 0.3$, which implies an emission altitude $\lesssim 1.3 R_{N S}$, which is the height where possibly also pair production could ensue. This altitude of emission agrees with the polar cap models (see e.g. Daugherty \& Harding 1996). A smaller energy cutoff, as in Harding et al. (1997), would have implied even lower emission altitude and a sharper break, possibly caused by the total absence of pair production. It is apparent that small differences in the emission position will cause strong differences in spectral shape. This is possibly the reason for the different emission properties of the two peaks as observed in the total (AGILE plus COMPTEL) gamma-ray energy band. Also, a trend can be observed, from lower to higher energies (see the X-ray light-curve for the trend in the fi rst peak, as in Fig. 3 of Kuiper et al. 1999), of the peaks slightly drifting away from the radio peak. This we assume to be another signature of the fact that small variations in emission height can be responsible for sensible changes in the light-curves in such a high magnetic fi eld. The scenario proposed by Harding et al. (1997) is strengthened by its prediction that PSR B0656+14 should have a cutoff with an intermediate value between PSR B1509-58 and the other gamma-ray pulsars. Additionally, PSR B1509-58 (Kuiper et al. 1999, Crawford et al. 2001) and PSR B0656+14 (De Luca et al. 2005, Weltevrede et al. 2010) show evidence of an aligned geometry, which could imply polar cap emission.

The polar cap model as an emission mechanism is debated. From the theoretical point of view, the angular momentum is not conserved in polar cap emission (Cohen \& Treves 1972, Holloway 1977, Treves et al. 2010). And a preferential explanation of the observed gamma-ray light-curves with high altitude cascades comes from the recent results by Fermi (Abdo et al. 2010a). In the case of PSR B1509-58, the derived gamma-ray luminosity from the flux at $E>1 \mathrm{MeV}$, considering a $1 \mathrm{sr}$ beam sweep is $L_{\gamma}=4.2_{-0.2}^{+0.5} d_{5.2}^{2} \times 10^{35} \mathrm{erg} / \mathrm{s}$, where $d_{5.2}$ indicates the distance in units of 
$5.2 \mathrm{kpc}$. While traditionally the beaming fraction $\left(f_{\Omega}\right)$ was considered to be the equivalent of a 1 sr sweep, nowadays (see e.g. Watters et al. 2009) the tendency is to consider a larger beaming fraction $\left(f_{\Omega} \approx 1\right)$, close to a $4 \pi \mathrm{sr}$ beam. Using $f_{\Omega}=1$ in our calculations, we would have obtained $L_{\gamma}=5.8_{-0.8}^{+0.1} d_{5.2}^{2} \times 10^{36} \mathrm{erg} \mathrm{s}^{-1}$. Thus the maximum conversion effi ciency of the rotational energy loss ( $\dot{E} \approx 1.8 \times 10^{37} \mathrm{erg} \mathrm{s}^{-1}$, see Section 1$)$ into gamma-ray luminosity is 0.3 . Our result is not easily comparable with the typical gamma-ray luminosities above $100 \mathrm{MeV}$, because for PSR B1509-58 this energy band is beyond the spectral break. Using AGILE data alone we obtained a luminosity above $30 \mathrm{MeV} L_{\gamma}=5.2(6) d_{5.2}^{2} \times 10^{35} \mathrm{erg} / \mathrm{s}$, again for a $1 \mathrm{sr}$ beam. If the gamma-ray luminosity cannot account for a large fraction of the rotational energy loss, then the angular momentum conservation objection from Cohen \& Treves (1972) becomes less cogent for this pulsar, exactly as it happens for the radio emission.

Alternatively, if such an effi ciency as that of PSR B1509-58 were incompatible with this conservation law, an interpretation of PSR B1509-58 emission can be sought in the frame of the three dimensional outer gap model (Zhang \& Cheng 2000). According to their model, hard X-rays and low energy gamma-rays are both produced by synchrotron self-Compton radiation of secondary $\mathrm{e}^{+} \mathrm{e}^{-}$pairs of the outer gap. Therefore, as observed, the phase offset of hard X-rays and low energy gamma-rays with respect to the radio pulse is the same, with the possibility of a small lag due to the thickness of the emission region. According to their estimates a magnetic inclination angle $\alpha \approx 60^{\circ}$ and a viewing angle $\zeta \approx 75^{\circ}$ are required to reproduce the observed light-curve. Finally, using the simulations of Watters et al. 2009), who produced a map of pulse profi les for different combinations of angles $\alpha$ and $\zeta$ in the different emission models, the observed light-curve from AGILE is best reproduced if $\alpha \approx 35^{\circ}$ and $\zeta \approx 90^{\circ}$, in the framework of the two pole caustic model (Dyks \& Rudak 2003).

The values of $\alpha$ and $\zeta$ required by the Zhang \& Cheng model are not in good agreement with the corresponding values obtained with radio measurements. In fact, Crawford et al. (2001) observe that $\alpha$ must be $<60^{\circ}$ at the $3 \sigma$ level. The prediction obtained by the simulations of Watters et al. (2009) better agrees with the radio polarization observations. In fact, in the framework of the rotating vector model (RVM, see e.g. Lorimer \& Kramer 2004 and references therein), Crawford et al. (2001) also propose that, if the restriction is imposed that $\zeta>70^{\circ}$ (Melatos 1997), then $\alpha>30^{\circ}$ at the $3 \sigma$ level. For these values, however, the Melatos model for the spin down of an oblique rotator predicts a braking index $n>2.86$, slightly inconsistent with the observed value ( $n=2.839$ (3), see Livingstone et al. 2005). Also in the case of PSR B0656+14, Weltevrede et al. (2010) conclude that the large values of $\alpha$ and $\zeta$ are somewhat at odds with the constraints from the modeling of the radio data and the thermal X-rays which seem to imply a more aligned geometry. Improved radio polarization measurements would help placing better constraints on the pulsar geometry and therefore on the possibility of a gap in the extended or outer magnetosphere, but the quality of the polarization measurements from Crawford et al. (2001) is already excellent, the problem being that PSR B1509-58, like most pulsars, only shows emission over a limited pule phase range and therefore the RVM models are highly degenerate.

At present the geometry privileged by the state of the art measurements is best compatible with polar cap models. Higher statistics in the number of observed gamma-ray pulsars could help characterize a class of "outliers" having gamma-ray emission from the polar caps, which potentially constitute a privileged target for AGILE. 


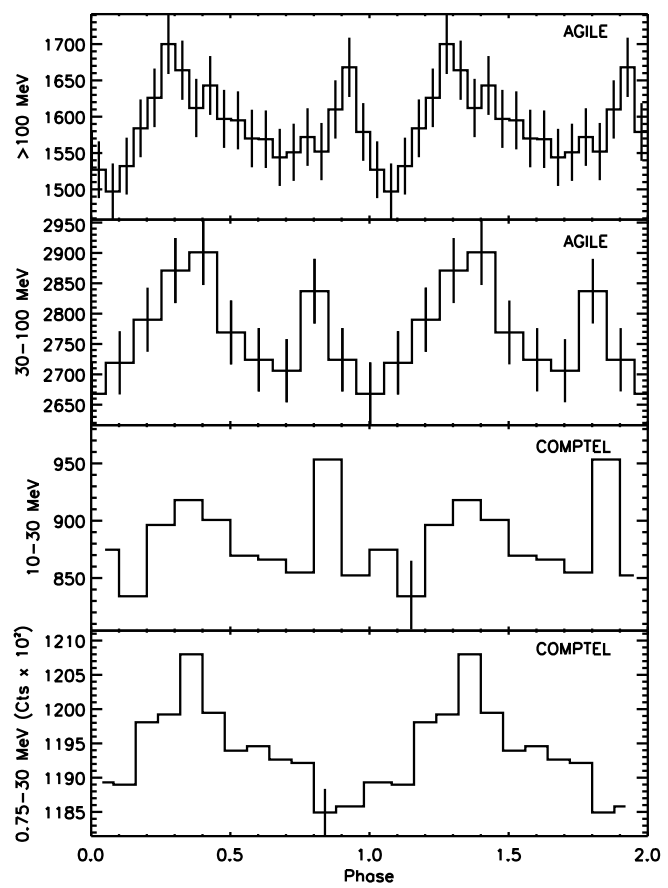

Figure 1: Phase-aligned gamma-ray light-curves of PSR B1509-58 with radio peak at phase 0. From the top: AGILE > $100 \mathrm{MeV}, 20$ bins, $7.5 \mathrm{~ms}$ resolution; AGILE $<100 \mathrm{MeV}, 10$ bins, $15 \mathrm{~ms}$ resolution; COMPTEL 10-30 MeV and COMPTEL 0.75-30 MeV (from Kuiper et al. (1999).

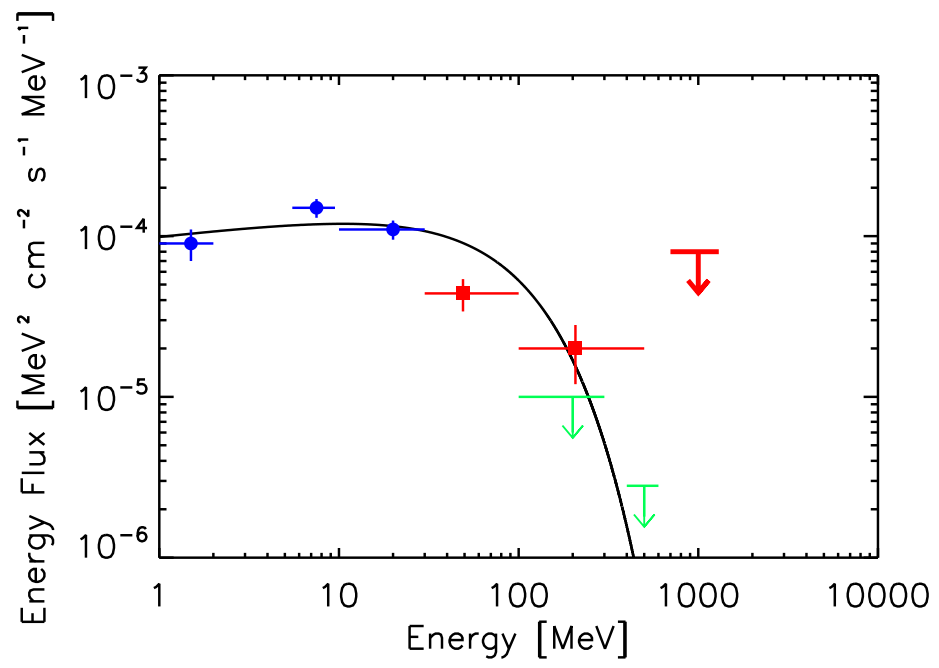

Figure 2: SED of PSR B1509-58 (solid line) obtained from a fit of pulsed fluxes from soft to hard gammarays. The three round points represent COMPTEL observations (Kuiper et al. 1999). The two square points represent $A G I L E$ pulsed flux in two bands $(30<E<100 \mathrm{MeV}$ and $100<E<500 \mathrm{MeV})$. The thicker arrow represents AGILE upper limit above $500 \mathrm{MeV}$. The two thin arrows represent Fermi upper limits from Adbo et al. (2010b) 


\section{References}

[1] Abdo, A. A. et al. 2010a, ApJS, 187, 460

[2] Abdo, A. A. et al. 2010b, ApJ, 714, 927

[3] Adler, S. L., et al. 1970, PhRevL, 25, 1061

[4] Cohen, R. H. \& Treves, A. 1972, A\&A, 20, 305

[5] Crawford, F., Manchester, R. N., \& Kaspi, V. M. 2001, AJ, 122, 2001

[6] Daugherty, J. K. \& Harding, A. K. 1996, ApJ, 458, 278

[7] De Luca, A., Caraveo, P. A., Mereghetti, S., Negroni, M., \& Bignami, G. F. 2005, ApJ, 623, 1051

[8] Dyks, J. \& Rudak, B. 2003, ApJ, 598, 1201

[9] Harding, A. K., Baring, M. G., \& Gonthier, P. L. 1997, ApJ, 476, 246

[10] Hobbs, G., Lyne, A. G., Kramer, M., Martin, C. E., \& Jordan, C. 2004, MNRAS, 353, 1311

[11] Hobbs, G. B., Edwards, R. T., \& Manchester, R. N. 2006, MNRAS, 369, 655

[12] Holloway, N. J. 1977, MNRAS, 181, 9P

[13] James, F. \& Roos, M. 1975, Computer Physics Communications, 10, 343

[14] Kuiper, L. et al. 1999, A\&A, 351, 119

[15] Livingstone, M. A., et al. 2005, ApJ, 619, 1046

[16] Lorimer, D. R., \& Kramer, M. 2004, Handbook of Pulsar Astronomy (Cambridge, UK: Cambridge University Press)

[17] Manchester, R. N., Tuohy, I. R., \& Damico, N. 1982, ApJL, 262, L31

[18] Melatos, A. 1997, MNRAS, 288, 1049

[19] Pellizzoni, A. et al. 2009a, ApJ, 691, 1618

[20] Pellizzoni, A. et al. 2009b, ApJL, 695, L115

[21] Pilia , M. et al. 2010, ApJ, 723, 707

[22] Tavani, M. et al. 2009, A\&A, 502, 995

[23] Treves, A., Pilia, M. \& Lopez, M. 2010, arXiv: 1011.6562

[24] Watters, K. P., Romani, R. W., Weltevrede, P., \& Johnston, S. 2009, ApJ, 695, 1289

[25] Weltevrede, P. et al. 2010, ApJ, 708, 1426

[26] Zhang, L. \& Cheng, K. S. 2000, A\&A, 363, 575 